\title{
Situación actual de la población adolescente en Costa Rica. Estudio realizado en centros educativos de Guanacaste, Puntarenas y Limón
}

\section{Current Situation of Adolescence in Costa Rica. Study Performed in Schools from Guanacaste, Puntarenas, and Limón}

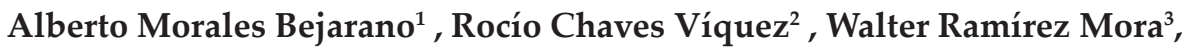 \\ Xiomara Jiménez Rodríguez ${ }^{4}$, Dalyana Vargas Acosta ${ }^{5}$, \\ Adriana García Borbón ${ }^{6}$, Isabel Yock Cabezas ${ }^{7}$
}

Clínica de Adolescentes, Hospital Nacional de Niños , Costa Rica

\begin{abstract}
Resumen. En 1991 y 2006, los profesionales de la Clínica de Adolescentes del Hospital Nacional de Niños realizaron dos investigaciones sobre población escolarizada de la provincia de San José, con el objetivo de identificar un perfil de adolescentes, dada la escasa información existente. Posteriormente, en el año 2013, se aplicó un instrumento similar a estudiantes de la Gran Área Metropolitana. En este artículo se presentan los resultados de la investigación desarrollada en 2015 con estudiantes de las provincias de Guanacaste, Puntarenas y Limón; se compararán los resultados previos para actualizar el estado de la situación integral de la población adolescente, explorando conocimientos y prácticas en sexualidad, violencia, consumo de drogas, nutrición, protección y riesgo. Se aplicó una encuesta a estudiantes de secundaria, hombres y mujeres, en edades comprendidas entre los 12 y los 18 años, en 31 colegios públicos y privados, para un total de 3074 estudiantes. Los resultados evidencian conocimientos deficientes en temas de desarrollo puberal y en información protectora en sexualidad; prácticas alimentarias de riesgo; incremento significativo en el consumo de drogas lícitas e ilícitas; exposición elevada a la pornografía; porcentaje bajo de inicio de actividad sexual coital; cifras altas de agresión, desesperanza e ideación suicida; elevado porcentaje de portación de armas, percepción positiva del papel del grupo de pares, la familia y el colegio. Asimismo, carencias en la formación integral y la adquisición de hábitos saludables, mediadas por la presión social que los promueve y debilidades significativas de sectores que deben brindar contención, apoyo y protección.
\end{abstract}

Palabras clave. Adolescencia, Sexualidad, Nutrición, Drogas, Riesgo, Salud

\begin{abstract}
In 1991 and 2006, professionals from the Adolescent Clinic of the National Children's Hospital conducted two investigations on the school population of the province of San José in order to identify a profile of adolescents, given the scarce information available. Subsequently, in 2013 a similar instrument was applied to students in the Great Metropolitan Area. This article presents the results of the research carried out in 2015 with students from the provinces of Guanacaste, Puntarenas and Limón and a comparison will be made with the previous results to update the status of the adolescent population's comprehensive situation, exploring knowledge and practices in sexuality, violence, drug use, nutrition, protection, and risk. A survey was applied to high school students, between the ages of 12 and 18, boys and girls from 31 public and private schools for a total of 3074 students. Results reveal the knowledge deficit on topics such as normal pubertal development and especially protective information on sexuality; unsafe feeding practices; significant increase of legal and illegal drug use, high exposure to pornography; low percentage of coital sexual activity onset, high rates of aggression, hopelessness and suicidal ideation, high percentage of weapon carrying, positive perception of the role of peers, family, and school. Also, deficiencies in the comprehensive training and the acquisition of healthy habits, mediated by the social pressure that promotes them and significant weaknesses of sectors that should provide containment, support and protection.
\end{abstract}

Keywords. Adolescent, Sexuality, Nutrition, Drugs, Risk, Health

\footnotetext{
${ }^{1}$ Médico pediatra, Universidad de Costa Rica. Jubilado; exjefe de la Clínica de Adolescentes, Hospital Nacional de Niños. Dirección electrónica: morabecr@ gmail.com

${ }^{2}$ Trabajadora Social, Universidad de Costa Rica. Dirección electrónica: mrchavesv@ccss.sa.cr; roskacr@hotmail.com

${ }^{3}$ Psicólogo clínico, Universidad de Costa Rica y Centro de Desarrollo Estratégico e Información en Salud y Seguridad Social (CENDEISSS). Dirección electrónica: ramirezwalter03@gmail.com

${ }^{4}$ Enfermera y terapeuta de familia, Universidad Libre de Costa Rica. Dirección electrónica: xiomarajimenezr@gmail.com

${ }^{5}$ Psicóloga clínica, Universidad Iberoamericana. Dirección electrónica: dalyanavargas@gmail.com

${ }^{6}$ Nutricionista, Universidad de Costa Rica. Dirección electrónica: agariabo@ccss.sa.cr

${ }^{7}$ Estadística y Administradora de Proyectos, Universidad de Costa Rica y Universidad Nacional. Dirección electrónica: iyockc@ccss.sa.cr
} 


\section{Introducción}

En los años 1991 y 2006, el personal de la Clínica de Adolescentes del Hospital Nacional de Niños realizó dos encuestas a población adolescente escolarizada, con el objeto de conocer e identificar factores de riesgo y de protección alrededor de temas sensibles que impactan directamente a este grupo etario.

Así, en 1991 se exploró sobre conocimientos y prácticas de adolescentes de secundaria en las áreas de desarrollo físico normal, higiene sexual, actividad sexual, abuso sexual, nutrición y alcohol y drogas en nueve colegios del área metropolitana.

Algunos de los datos obtenidos más relevantes mostraron un inicio temprano, antes de los quince años, de conductas de riesgo: actividad sexual, uso de tabaco, alcohol y otras drogas. Además, este se asocia a conocimientos limitados sobre sexualidad.

En el mismo sentido, se demostró una correlación alta y positiva entre conductas de riesgo, por ejemplo, primera relación sexual e inicio de consumo de alcohol, tabaco y otras drogas.

Aunado a ello, una alta vulnerabilidad al abuso sexual y a su perpetuación por la indefensión en cuanto a conocimientos correctos de las circunstancias del abuso y responder ante este.

Con respecto a su nutrición, los resultados evidenciaron la necesidad de ofrecer una adecuada educación nutricional a los adolescentes, ya que, tanto conocimientos como prácticas, evidentemente eran insuficientes e inadecuados.

Por otro lado, en el año 2006, se efectuó una encuesta similar a la de 1991 en un total de diez colegios del área metropolitana, en los temas de desarrollo físico, sexualidad, violencia, nutrición, consumo de alcohol, tabaco y drogas ilícitas; otras conductas de riesgo y protección. En ella, se evidenciaron nuevamente pocos avances en conocimientos y prácticas protectoras.

Dado que estas investigaciones enfatizaron sobre importantes carencias en conocimientos protectores que posee la población adolescente, así como prácticas sociales cada vez más desinformadas, surge la necesidad de conocer contemplar cómo se encuentran otras zonas del país. Por ello, el equipo profesional de la Clínica de Adolescentes, con apoyo del Centro de Informática y Estadística del Hospital Nacional de Niños, decidieron actualizar en los años 2013 y 2015, el estado de la situación de estos aspectos en población escolarizada, y a la vez plantear alternativas de intervención para fortalecer conocimientos protectores, prácticas seguras y opciones esperanzadoras ante situaciones de adversidad.

Para el 2015, el objetivo general de la investigación fue realizar una actualización diagnóstica acerca de conocimientos y prácticas de personas adolescentes que cursan educación secundaria en colegios de las provincias de Guanacaste, Puntarenas y Limón, en los temas de sexualidad, violencia en general, nutrición, consumo de alcohol, tabaco y otras drogas, así como otras conductas de riesgo y protección.

\section{Metodología}

\section{Población de estudio}

Estudiantes de secundaria que cursan de sétimo a duodécimo año en colegios diurnos, ya sean públicos, privados, técnicos o científicos de las provincias de Guanacaste, Puntarenas y Limón en el año 2015.

Tamaño de muestra

$p=0.5$ Porcentaje de adolescentes con conocimientos sobre aspecto varios

$D=.08$ Máximo error permisible 
$\mathrm{Z} \alpha \frac{1 / 2}{2}=1.15$ Valor de la curva normal, con una probabilidad de un $75 \%$

$N=316$, Colegios ubicados en la Gran Área Metropolitana

$$
n=\left[\frac{1.15^{*} \sqrt{0.5^{*}} 0.5}{0.09}\right] * * 2=62
$$

$10 \%$ de no respuesta $n=61 \mathrm{~N}$ corregida por población infinita: 36

\section{Selección de la muestra}

- Los colegios se agruparon según listado por provincia, el cual fue proporcionado por el Departamento de Estadística del Ministerio de Educación Pública de Costa Rica.

- Tomando el tamaño de muestra calculado anteriormente, se eligieron por provincia y un número proporcional de colegios ubicados en Guanacaste, Puntarenas y Limón, según su cantidad en cada provincia y tipo (privado, público). El tamaño de la muestra se calculó tratando de encuestar de 30 a 40 colegios aproximadamente, debido a la carencia de recursos humanos y financieros para obtener una muestra de colegios más amplia. De esta forma, se pretende alcanzar al menos 3300 estudiantes encuestados.

- En cada una de las regiones, se les asignó un número consecutivo a cada colegio.

- De cada provincia, se seleccionó proporcionalmente $\mathrm{n}$ cantidad según el total de colegios reportados.

- La selección de los colegios se llevó a cabo de forma sistemática.

- Debido al número variable de alumnos y grupos en cada uno de los colegios seleccionados, sumada a la imposibilidad material de entrevistar la totalidad de los grupos de $7 .^{\circ}$ a $12 .^{\circ}$ año en cada institución, se predeterminó el siguiente procedimiento para elegir los grupos para encuestar:

o El total de encuestas por repartir era de aproximadamente 4500, para alcanzar al menos 3300 alumnos encuestados, tomando en cuenta la tasa de deserción de un $10,7 \%$ y de exclusión de un $16 \%$.

o Lo anterior es un aproximado, dado que no se podía tener con exactitud la cantidad de alumnos que, al momento de la entrevista, estaría presente en cada aula.

o De cada nivel se seleccionó un grupo. Docentes a quienes se les asignó la tarea de pasar la encuesta, seleccionaron el primer grupo del respectivo nivel al que le impartió clase, después de haberles entregado las encuestas.

o Una vez escogido el grupo de cada nivel, se encuestó la totalidad de los alumnos de cada aula.

- En los colegios que tenían únicamente uno de los niveles, se entrevistó a todo el estudiantado de ese único nivel.

- La entrevista fue de tipo autoadministrado, es decir, se les dio una introducción y se le entregó un cuestionario a cada estudiante, con el fin de que lo llenaran en forma anónima.

- Los paquetes de cuestionarios se organizaron por colegios y, dentro de estos, por aulas para cada nivel.

- La selección del número de aulas, para efecto deenviarel material, secalculóaproximadamente de acuerdo con el número de alumnos por nivel de cada colegio. Bajo el supuesto de que cada aula en los colegios públicos tiene un promedio de 25 .

- Los aspectos por investigar, así como las preguntas elaboradas en la encuesta, fueron resultado de un análisis cuidadoso del equipo profesional en cuanto a los ítems sobre los que 
se quería obtener conocimientos, según los objetivos de la investigación y basados en las encuestas de las investigaciones anteriores.

\section{Resultados}

\section{Caracterización de la población}

Del total de 3074 adolescentes entrevistados, $51 \%$ son femeninos, $45 \%$ masculinos y $4 \%$ no responden (Figura 1).

La distribución por nivel educativo se puede ver en la Figura 2.
El porcentaje de repitencia en la población es de un $22 \%$, siendo sétimo y octavo año los niveles con mayor porcentaje de repitencia.

De la población estudiada, el 55\% viven con ambos padres, el $22 \%$ solo conla madre, el $13 \%$ con la madre y padrastro, y $6 \%$ con otros familiares.

Prácticas y conocimiento en sexualidad

Según la Organización Mundial de Salud (2016), la salud sexual es un estado de bienestar físico, mental y social; requiere un enfoque

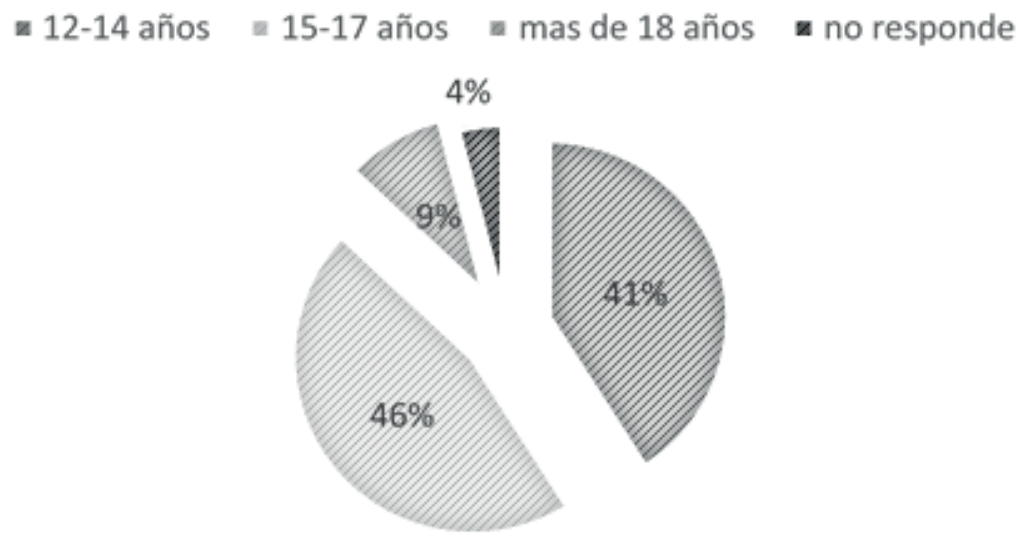

Figura 1. Estudiantes entrevistados según edad.
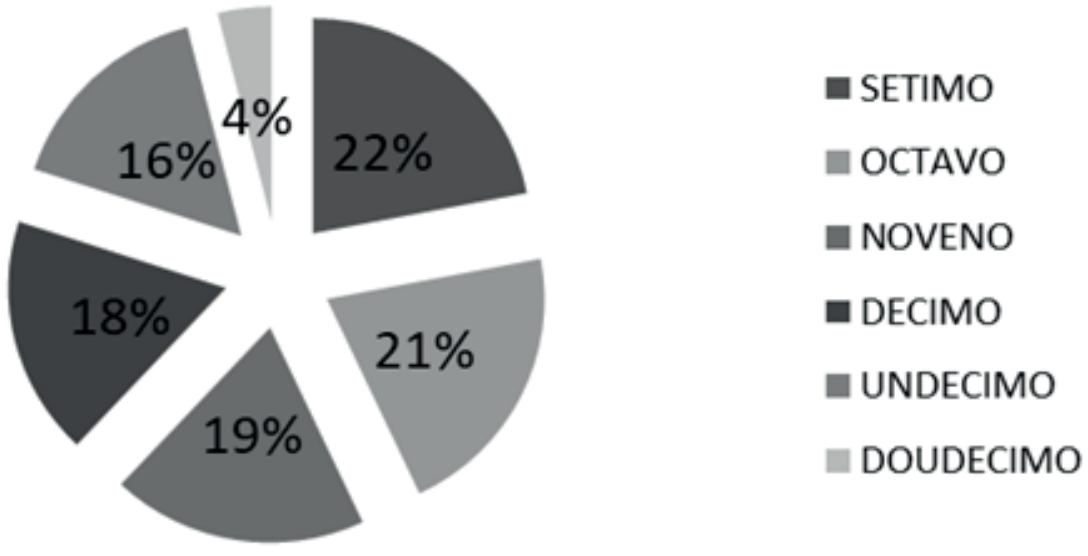

Figura 2. Estudiantes entrevistados según nivel educativo 
positivo y respetuoso de la sexualidad y de las relaciones sexuales, así como la posibilidad de tener experiencias sexuales placenteras y seguras, libres de toda coacción, discriminación y violencia.

Partiendo de que la población adolescente se encuentra en un periodo formativo, deberían estar recibiendo una adecuada preparación para vivir una sexualidad plena. Empero, los datos encontrados no reflejan esta formación integral.

Refiriéndose a la masturbación, el porcentaje de personas adolescentes que lo consideran un evento normal es de un $40 \%$, un $18 \%$ mantienen mitos al respecto y un $42 \%$ responde no saber.

Para el $40 \%$ la masturbación es un evento normal, este porcentaje es menor si se compara con las encuestas anteriores. Lo que representa que más de la mitad de los encuestados maneja mitos respecto a este suceso, demostrando conocimientos de sexualidad deficientes y poca información veraz.

En cuanto a información protectora, la cantidad de adolescentes que conocen los días fértiles de la mujer es de un $17 \%$, un $30 \%$ da respuestas incorrectas y el 53\% no saben.

Datos similares se observaron en la SJ06 (Investigación realizada en provincia de San José en el 2006), 17\% y 21\% para la encuesta del GAM13 (Investigación realizada en Gran Área Metropolitana en el 2013). Este aumenta significativamente si se compara con lo reflejado en SJ91 (Investigación realizada en provincia de San José en 1991), que fue de un 7\%, lo cual puede estar asociado al acceso a la información a través de diversos medios.

Cuando se pregunta si hay riesgo de embarazo cuando el hombre saca el pene antes de eyacular, encontramos respuestas correctas en un 35\%, incorrectas en un 33\%, no saben el $29 \%$ y un $3 \%$ no responde. Estos datos son similares a las encuestas anteriores.
En relación con los conocimientos para prevenir infecciones de trasmisión sexual, un $20 \%$ conoce las medidas adecuadas, un $70 \%$ maneja información parcial y un $10 \%$ no sabe la respuesta. Datos similares a los encontrados en la Gran Área Metropolitana, pero mucho menor a la encuesta del 2006 que era de un 35\%.

Sobre la pregunta de si el VIH se puede trasmitir por medio del sexo oral, el $49 \%$ responde correctamente, el 19\% incorrectamente, un $27 \%$ no sabe y un 5\% no responde.

A la pregunta de si el virus del papiloma humano se puede transmitir aun utilizando preservativo, el 35\% contestó correctamente, incorrectamente el $18 \%$, no sabe el $42 \%$, no responde un $5 \%$.

A la consulta de si las pastillas anticonceptivas deben tomarse todos los días, aunqueno se tengan relaciones sexuales, contestó correctamente el $62 \%$, incorrectamente el $8 \%$, no sabe el $27 \%$ y no responde el 3\%.

Por último, sobre si se puede quedar embarazada si el hombre roza con el pene la entrada de la vagina sin preservativo, respondió correctamente el 34\%, incorrectamente el 32\%, no sabe el $30 \%$ y no responde el $4 \%$.

En todos los resultados, las consultas son poco alentadoras y confirma el riesgo que conlleva el desconocimiento de temas elementales que deberían ser comunicados y discutidos en los centros de educación, salud y en las mismas familias.

Esta información confirma la exposición de la población adolescente a actividad sexual de riesgo, dando como resultado que no se logre un desarrollo pleno, sano y seguro de su vida sexual, constituyendo un desafío para los interesados en la salud de los adolescentes.

Los adolescentes experimentan con varios roles en la búsqueda de su identidad; esto les permite 
adoptar conductas de salud, ya sean promotoras de la salud o de riesgo. Respecto a las prácticas, un $26 \%$ de los adolescentes encuestados había mantenido relaciones sexuales coitales, es el dato más alto comparado con las encuestas anteriores.

En todos los casos, la mayoría de los encuestados no ha iniciado la actividad sexual coital, podríamos suponer que la permanencia dentro del sistema educativo formal se convierte en un factor protector para los adolescentes.

En cuanto a la edad de inicio de la actividad sexual, se puede observar la distribución en la Figura 3.

A partir de la encuesta SJ06, la edad de inicio de la actividad sexual coital se mantiene entre los 13 y los 15 años como promedio, lo que es importante de destacar dado que la educación sexualimpartida demanera formalen los colegios de secundaria del país inicia en sétimo año, o sea, cuando la mayoría de adolescentes tiene 13 años; por lo tanto, se debería tomar en cuenta la oportunidad que significaría abordarla antes de que los jóvenes inicien relaciones sexuales, es decir, en los años escolares entre los 10 y los 12 años, cuando cursan quinto y sexto grado. En busca de mejorar sus conocimientos previos.

Es necesario mencionar que los casos de actividad sexual, antes de los 12 años, constituyen abusos sexuales.

Se nota un cambio importante entre la encuesta de SJ91 y las subsiguientes en cuanto a edad de comienzo de la actividad sexual, dado que esta disminuyó en dos años.

De los adolescentes que iniciaron su actividad sexual, encontramos que usaron métodos anticonceptivos, según se explica en la Figura 4.

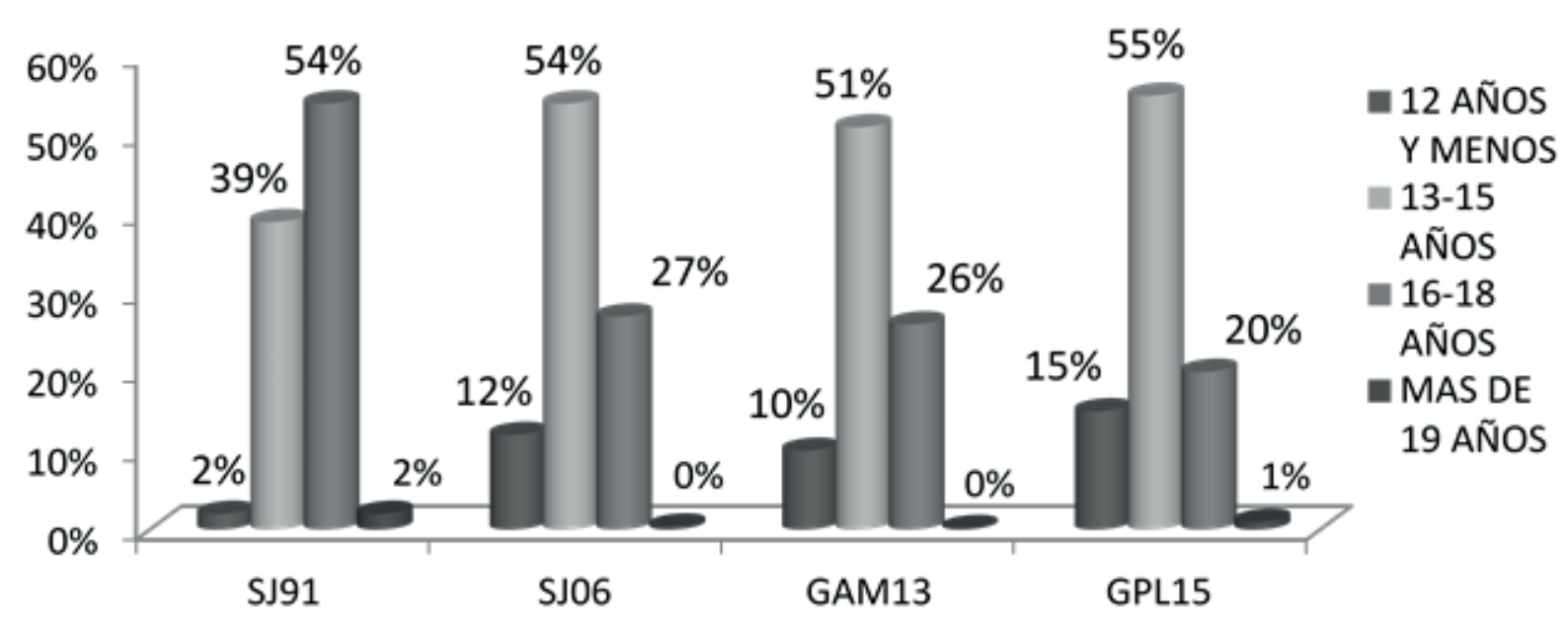

Figura 3. Estudiantes entrevistados según edad de inicio de actividad sexual. SJ91: Investigación realizada en provincia de San José en 1991; SJ06: Investigación realizada en provincia de San José en el 2006; GAM13: Investigación realizada en Gran Área Metropolitana en el 2013; GPL15: Investigación realizada en Guanacaste, Puntarenas y Limón en el 2015. 


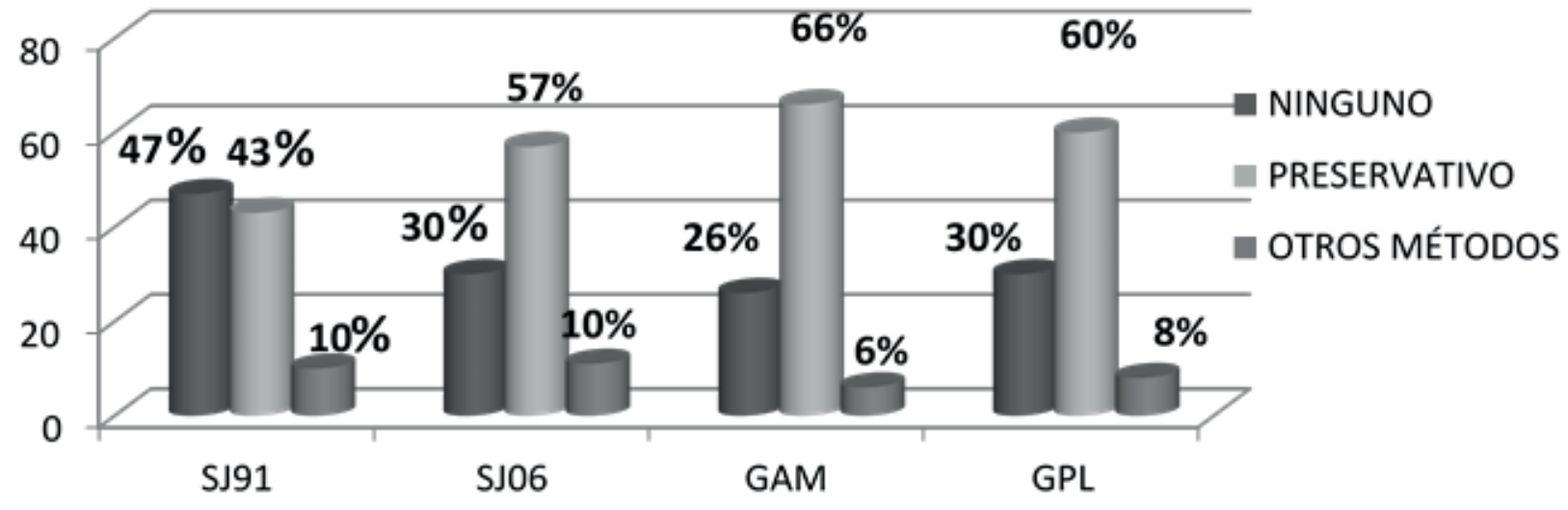

Figura 4. Estudiantes entrevistados según método anticonceptivo utilizado. SJ91: Investigación realizada en provincia de San José en 1991; SJ06: Investigación realizada en provincia de San José en el 2006; GAM13: Investigación realizada en Gran Área Metropolitana en el 2013; GPL15: Investigación realizada en Guanacaste, Puntarenas y Limón en el 2015.

Se evidencia en estos datos que, a partir de la encuesta del año 1991, el uso del preservativo es mucho más frecuente entre los adolescentes, lo que se constituiría en un paso de protección importante, en materia de prevención de infecciones de trasmisión sexual (ITS), pero no tantoenlaprevencióndeunembarazonoplaneado.

El uso o no del preservativo puede incidir en otros aspectos tales como su disposición, el precio de compra, facilidades de uso, y la información difundida respecto a su utilidad.

De igual manera, los porcentajes de personas adolescentes que no usaron ningún método de anticoncepción continúa siendo muy alto, lo que podríamos relacionar con el porcentaje de maternidad en adolescentes que fue de un $8 \%$ para el 2015.

Por lo tanto, entre las personas adolescentes, el porcentaje de uso de otros métodos anticonceptivos es bajo, lo que podría deberse a la poca información de la que disponen y a la dificultad de esta población para acceder a servicios de salud amigables, que aborden el tema sin prejuicios y con personal capacitado.

Lo anterior cuestiona el tipo de educación sexual impartida en los hogares y el tiempo que las personas adultas dedican para dialogar y conversar con las personas menores de edad. Las personas adultas deben regular, de alguna manera, la tecnología que se pone a disposición de las personas menores de edad. Se requiere una comunicación asertiva de las personas cuidadoras hacia las y los menores, con la cual se fomente la validación de sentimientos y no se juzguen las actitudes y conductas.

En cuanto al acceso a pornografía, un 50\% de las personas adolescentes han tenido exposición. De este porcentaje, en el $22 \%$, la exposición se 
da entre los 5 y 11 años de edad y un 57\% entre los 12 y 15 años. El medio más utilizado por la población para tener acceso fue el teléfono celular en un $84 \%$ de los casos, un $35 \%$ videos y un $30 \%$ en la televisión.

Respecto a la edad promedio de la menarca (primera menstruación) es de 12 años, 11 meses; en la investigación del GAM13, el promedio era de 12 años 5 meses.

\section{Exposición y conocimientos en violencia}

La información se presenta en dos momentos: el primero contiene el conocimiento de la población adolescente entrevistada en relación con lo que consideran es abuso en todas sus manifestaciones, y el segundo momento muestra quiénes han sido sobrevivientes de este.

Para ello, la población identificó como abuso sexual (Tabla 1), lo siguiente:

Tabla 1

Tipología de abuso sexual según criterio de adolescentes

Decirle vulgaridades a una persona $5 \%$

Ver pornografía de forma obligada

$12 \%$

Obligar a una persona menor de edad a ser fotografiada desnuda

$19 \%$

Tocar los genitales sin consentimiento

$20 \%$

Involucrar a una persona menor de edad en explotación sexual

$22 \%$

Incesto

$24 \%$

Violación

$31 \%$

Todas las anteriores

$53 \%$

En cuanto a los conocimientos sobre el tema de violencia, más de la mitad de los adolescentes encuestados reconocen que el abuso sexual se constituye por diferentes prácticas tales como tocamientos, violación, incesto, obligar a otros a ver pornografía, entre otros. Si tomamos en cuenta que para la OMS (2013), el abuso sexual “(...) abarca actos que van desde el acoso verbal a la penetración forzada y una variedad de coacción, desde la presión social y la intimidación a la fuerza física" (p.1), todavía hay una cantidad importante de jóvenes que no reconocen en un tocamiento o en decir vulgaridades un acto de abuso sexual, esto por la falta de educación que aun no se da correctamente en los hogares y en los centros educativos. Es necesario no solo "enseñar", sino también sensibilizar en el tema del abuso. Este dato de poco más del $50 \%$ es similar en las encuestas anteriores.
Con respecto a las personas agresoras, en el tema general de violencia, el $49 \%$ de adolescentes responde que son personas desconocidas quienes cometen las ofensas, mientras que un $23 \%$ menciona que son conocidas, y un $23 \%$ dice no saber.

Como arrojan los datos, la mitad de las personas adolescentes encuestadas reconoce que son desconocidos quienes comenten más abusos sexuales, esta información no es del todo correcta, porque, según el conocimiento previo adquirido por los profesionales de la Clínica, son las personas cercanas y conocidas las que tienen mayor acceso a las personas menores de edad y quienes más cometen todo tipo de abusos. Asimismo, un $75 \%$ menciona que una persona menor de edad sí puede agredir sexualmente. 
Por otro lado, un $70 \%$ considera que los insultos, gritos y comparaciones son expresiones de abuso emocional, y un $79 \%$ manifiesta que los golpes entre miembros de la familia no son formas de resolver los conflictos.

Otros datos que señalan mayor conocimiento de la población encuestada sobre violencia reflejan que sí creen que las personas menores de edad pueden cometer ofensas sexuales. Aunado a esto, consideran que los gritos o comparaciones son abuso emocional y más del $70 \%$ menciona que en la vida cotidiana los golpes son formas de resolver conflictos. Estos datos son coherentes con las investigaciones anteriores, por ello se puede identificar que existen percepciones negativas con respecto a la violencia.

Al preguntárseles si la violación ocurre porque las mujeres provocan a los hombres, un $58 \%$ responde que no, sin embargo, un $15 \%$ si lo consideran así, un 19\% manifiesta no saber y un $8 \%$ no responde. Este dato es mucho menor a los obtenidos en el estudio GAM13 y SJ06, que eran de un $73 \%$ y $75 \%$, respectivamente.

Un $12,5 \%$ de las personas encuestadas ha sufrido algún tipo de agresión. De estos, el abuso emocional alcanza un $61 \%$, seguido del físico $34 \%$ y sexual $24 \%$. El tipo de abuso padecido puede verse comparativamente en la Figura 5, en relación con todos los estudios realizados.

El mayor porcentaje dice ser víctima de abuso emocional, seguido del abuso físico y luego el sexual. Es menester que las personas adolescentes reconozcan el abuso emocional, pues se tienden a minimizar sus efectos o a verlo como parte de la relación natural entre las personas. Sin embargo, estos datos evidencian que para los encuestados es claro que es un tipo de abuso importante, probablemente debido a

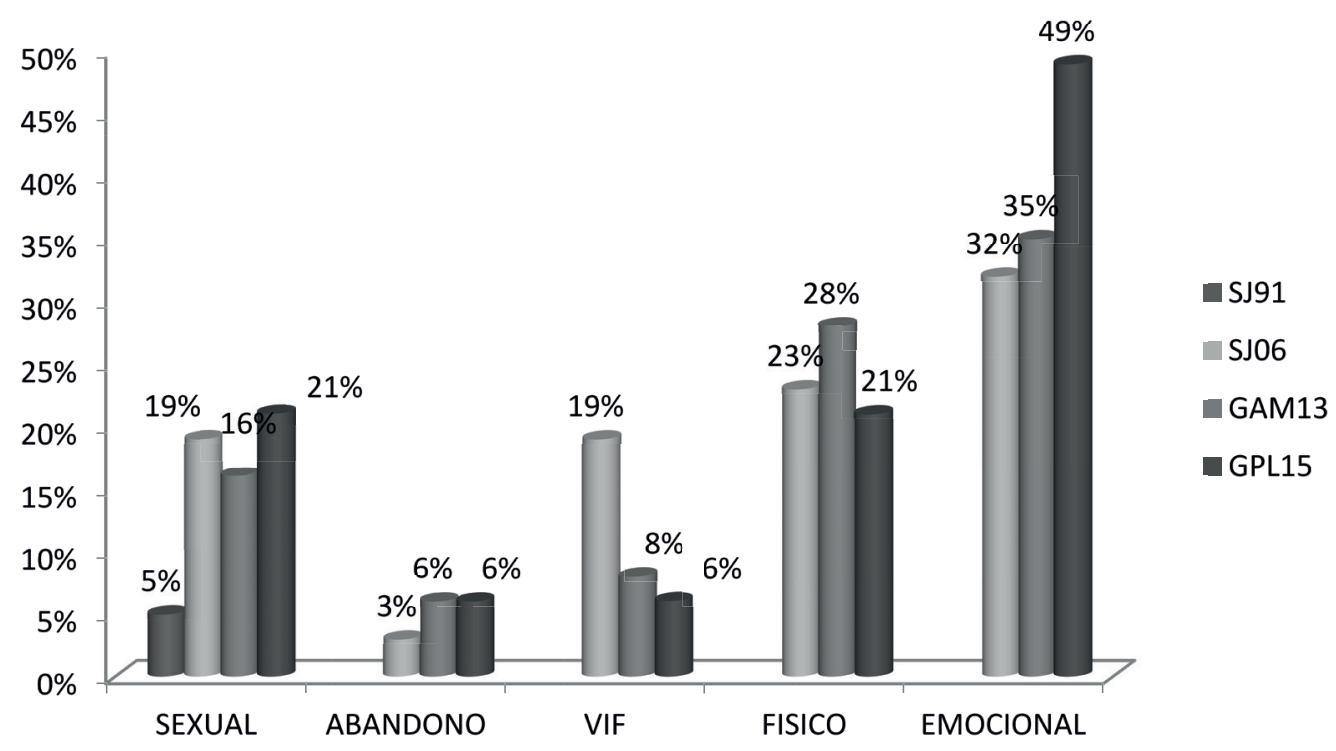

Figura 5. Estudiantes entrevistados según tipo de abuso del que fueron objeto. 
las campañas de información y sensibilización respecto al tema.

El $57 \%$ responde que quienes le agredieron fueron familiares, siendo el padre y la madre quienes ocupan el primer lugar (32\%), seguidos de hermanos, padrastros, tíos, primos, entre otros. Con respecto a las personas agresoras, más de la mitad refiere que fueron familiares quienes los abusaron, lo cual se contrapone a lo respondido anteriormente acerca de que las personas desconocidas son quienes cometen más abusos.

Un $28 \%$ responde que quienes le agreden son compañeros o compañeras del colegio o amistades, refiriéndose a el bullying que ha empezado a ser más documentado en los centros educativos del país. Es necesario que las autoridades educativas tomen este fenómeno con seriedad en tanto este tipo de violencia puede afectar significativamente el desempeño académico y emocional de los estudiantes, dejando secuelas permanentes en el tiempo. Por último, un $7 \%$ refiere que sus agresores fueron personas particulares.

La distribución de los porcentajes anteriores se encuentra de manera similar en las encuestas de 2006 y 2013, resaltandoquesonlospadresymadres quienes cometen mayor cantidad de abusos.

En la Tabla 2, se aprecian los lugares donde se cometió el abuso.

Tabla 2

Distribución de los lugares en los que ocurrió el abuso

\begin{tabular}{lc}
\hline Casa de la persona agresora & $15 \%$ \\
Colegio & $20 \%$ \\
Vía pública & $5 \%$ \\
Casa de la víctima & $49 \%$ \\
\hline
\end{tabular}

En cuanto a los lugares donde ocurrieron los episodios de abuso, predomina la casa de la víctima, lo cual es congruente con que los ofensores sean familiares y cercanos, donde más se dan estas situaciones es la casa de las víctimas que muchas veces comparten con sus ofensores. Esta combinación de factores atenta contra la prevención y la protección de adolescentes y deja de manifiesto que el fenómeno de la violencia está presente en las familias y se mantiene bajo secreto, con el riesgo que conlleva.

Siguiendo con el tema de la violencia que enfrenta nuestro país y que deben vivir las personas adolescentes cotidianamente, se preguntó en el estudio sobre el uso de armas, tanto en el ámbito educativo como en la vía pública. Las respuestas a las preguntas planteadas revelaron que el $4 \%$ ha portado armas en el colegio; aunque en la calle las ha portado un porcentaje mayor de jóvenes, $9 \%$. Estos datos presentan un aumento en cuanto a los datos arrojados para la GAM que era de un 3 y $6 \%$, respectivamente; en el 2006, los datos arrojaron un 7 y $11 \%$, siendo los más altos. De quienes han llevado armas al colegio, un $64 \%$ son hombres y $32 \%$ son mujeres. En la calle, el porcentaje de hombres que ha portado armas es de un $69 \%$ y mujeres de un $26 \%$.

Evidentemente, la presencia de armas cerca de nuestros jóvenes ha aumentado y se constituye como un factor de riesgo, especialmente en 
aquellos casos donde se evidencian signos y síntomas de problemas emocionales (Hernández, Sánchez, y Cañón, 2013). Es necesario dar a conocer a las personas adolescentes el riesgo que conlleva la manipulación de armas y los daños permanentes que puede ocasionar su uso. Además de profundizar en los motivos que llevan a los adolescentes a utilizarlas.

\section{Consumo de drogas}

De acuerdo con los resultados obtenidos, un $19 \%$ ha fumado y un $51 \%$ ha consumido licor alguna vez en la vida; un 5\% de la población ha fumado y un $19 \%$ ha consumido bebidas alcohólicas en los últimos treinta días y un 21\% ha tenido una conducta de embriaguez. El 11\% reporta haber consumido otro tipo de drogas. La distribución por sexo se muestra en la Figura 6.

El inicio de la edad de consumo de tabaco, en su mayoría, se da entre 11 y 14 años en un $58 \%$ de la población encuestada, un $24 \%$ entre 15 años o más y $12 \%$ entre los 7 y 10 años.
En cuanto al tema de adicciones, en el 2015, en la encuesta realizada por el Instituto de Alcoholismo y Farmacodependencia (IAFA), se encontró que la edad de inicio del fumado se da en una media para las mujeres de 13,7 años y para los varones es de 13,4 años. Datos similares se encontraron en este estudio, en que la conducta de fumado inicia entre los 11 y 14 años para el $64 \%$ de los adolescentes entrevistados y la ingesta etílica en un 63\% entre los 10 y 14 años.

En relación con la edad de inicio del consumo de licor, el 63\% lo hace entre los 10 y 14 años, el $25 \%$ a los 15 años o más y el $8 \%$ entre los 5 y 9 años. Para el consumo de alcohol se tiene que la edad promedio de inicio es a los 13 años en la investigación del GAM13. Pese a ello, según los datos obtenidos en la encuesta realizada por IAFA (2015), los porcentajes han ido en aumento desde el año 2009.

La edad de inicio en el consumo de otras drogas es entre los 12 y 15 años para el 65\%, el $10 \%$ entre los 7 y 11 años, y un 16\% más de 16 años. La marihuana es la droga más utilizada por

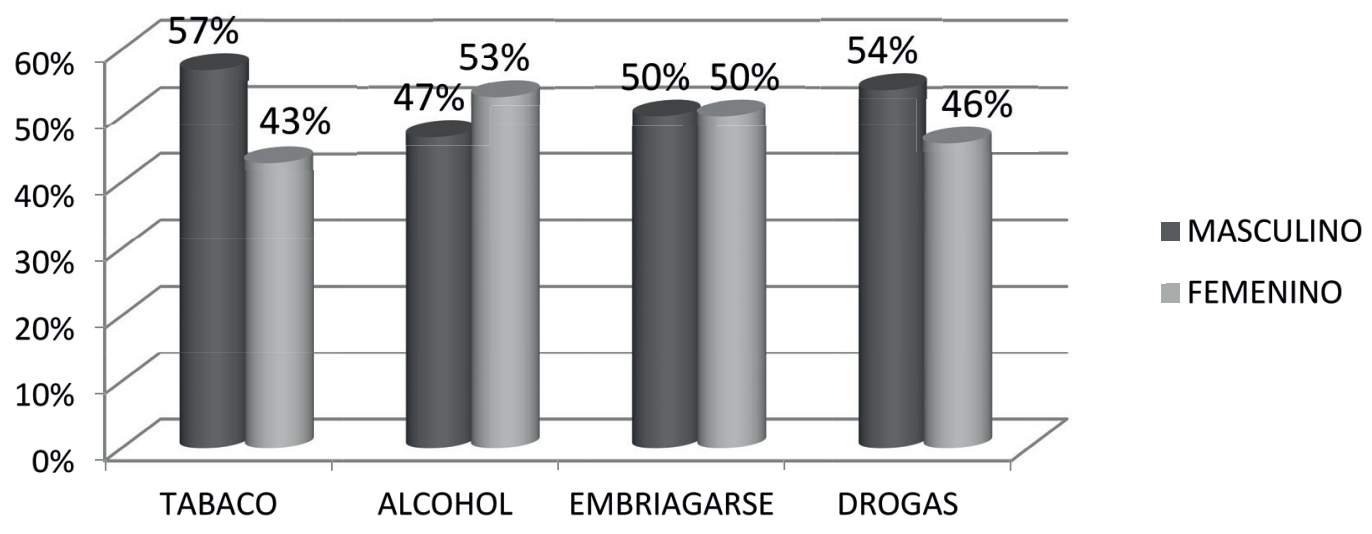

Figura 6. Estudiantes entrevistados según consumo. 
los adolescentes, para un 90\%, le sigue la cocaína en un $16 \%$, el éxtasis en un $11 \%$, el cemento en un $9 \%$ y el crack en un $8 \%$.

Por otro lado, ante la consulta sobre el uso de otras sustancias adictivas (marihuana, cocaína, crack, heroína, entre otras), se obtuvo un 15\% de respuestas positivas, lo cual representa un aumento del $5 \%$ en comparación con la encuesta realizada en 2006 y 14\% que en SJ91. De la misma forma, según los datos del IAFA (2015), se muestra un incremento en el uso de dichas sustancias, pasando del 14,7\% en 2006 a 16,6\% en 2012.

Según la última encuesta de IAFA (2015), prevalece el consumo de marihuana del 9,4\%, en este estudio se obtuvo que el $11 \%$ usó algún tipo de droga y de este porcentaje, el 78\% utilizó marihuana, siendo menor al porcentaje de la encuesta del GAM13 (87\%). La edad de inicio ronda los 14 años, aproximadamente.

De la cantidad de adolescentes que afirmaron haber consumido otras drogas, se encontró que un $7 \%$ manifiesta haber consumido crack y cocaína, mientras que en la encuesta del GAM13 fue de un 5\%. Asimismo, el $4 \%$ utilizó éxtasis y un 3\% lo reportó en la encuesta GAM13.

Toda esta información deja ver que sigue en aumento el consumo de alcohol y otras drogas, sin embargo, también se nota la disminución en el consumo de tabaco, lo que podría deberse a las campañas preventivas de uso de tabaco.

\section{Aspectos nutricionales}

En cuanto a los temas de alimentación existen gran cantidad de documentos los cuales nos indican que el desayuno es una de las comidas más importantes del día, la encuesta nos muestra que un $71 \%$ de adolescentes realiza este tiempo de comida, pero, en relación con la encuesta anterior, no hay una mejoría importante. En un $80 \%$, los jóvenes comen acompañados, lo cual representa un alto porcentaje y supera al GAM; esto se convierte en un hábito protector para la población adolescente.

El $37 \%$ indica que ve revistas o programas de moda y belleza frecuentemente, esto ha persistido en comparación con la encuesta anterior y podría mostrar que las herramientas para ver este tipo de información ya no son las mismas. No obstante, continúa siendo un potencial riesgo la obsesión por la belleza y el cuerpo, lo que podría aumentar la probabilidad de tener un trastorno de la alimentación, incluso porque el $28 \%$ de los jóvenes hace dietas para perder peso y el $7 \%$ reporta haber utilizado algún medicamento con este fin.

En cuanto al consumo de comidas rápidas, el $10 \%$ las ingiere todos los días, el $15 \%$ tres veces por semana, el $26 \%$ al menos una vez por semana. Cada 15 días el 13\%, una vez al mes $25 \%$ y nunca un $7 \%$.

El hecho de que el $26 \%$ de adolescentes incluye al menos una vez por semana el consumo de comida rápida es un dato que se ha mantenido respecto a las encuestas anteriores, mostrando que el problema continúa a lo largo de los años, con la posibilidad del aumento de las enfermedades crónicas en la población joven.

Frente al crecimiento acelerado del consumo de comidas rápidas en la población adolescente, resulta necesario indagar el tema del ejercicio físico en dicha población. El 16\% reporta no hacer ejercicio, el $34 \%$ hace ejercicio una vez por semana, el $20 \%$ tres veces por semana, el $23 \%$ todos los días.

Ante ello, en la presente investigación se tiene que el $34 \%$ de las personas encuestadas menciona realizar algún tipo de ejercicio una vez por semana, esto hace pensar que es la clase de educación física que se da semanalmente en escuelas y colegios, por tanto, hay una población considerada como sedentaria, al igual que en la encuesta anterior con un $53 \%$. 
Percepción, protección y relaciones

Según los resultados obtenidos en esta área, con respecto a la percepción de las personas adolescentes sobre el apoyo que reciben, el 85\% afirma sentirse apoyado en sus hogares y el $72 \%$ en el colegio.

La percepción de la población adolescente sobre el apoyo que reciben en cada uno de sus hogares y el colegio se consideran factores protectores ante las circunstancias que viven diariamente, en esta investigación se puede notar que los jóvenes encuestados, en su gran mayoría, tienen hogares e instituciones educativas que son fuentes de seguridad y protección, que los ayudan en su crecimiento y desarrollo.

Datos muy similares se evidenciaron en las investigaciones hechas en el 2006 y 2013 que abarcan a los adolescentes de la Gran Área Metropolitana, donde se obtuvieron 83\% y $89 \%$, respectivamente, que percibían apoyos familiares y un 71\% en el 2006, y en el 2013 un $75 \%$ percibían a las instituciones educativas como recursos de apoyo.

Estos datos destacan que las familias costarricenses y los colegios tienden a ser fuentes invaluables proveedoras de valores y civismo, los cuales potencien la conciencia moral y cultural de los adolescentes. Tal y como lo describen Soler, Pérez y Mejías (2016), se deben fortalecer los vínculos familiares, ya que permite a la juventud tener mayores recursos de apoyo que favorezca la búsqueda de solución de problemas y se prevengan conductas impulsivas y de riesgo. Además, Rivera y Chuana (2016) mencionan que un adecuado funcionamiento familiar, donde existan relaciones estables, con miembros unidos que se adaptan a los cambios, se encuentran satisfechos con su familia y tienen una comunicación fluida entre padres e hijos, disminuyen la probabilidad de presentar conductas antisociales.
En relación con los deseos de morir y los planes analizados por los jóvenes para acabar con su vida, se encontró que un $15 \%$ de los encuestados ha tenido deseos de morir y el 10\% ha elaborado un plan para llevarlo a cabo.

Estos datos relevantes ponen en alerta sobre la situación actual, nos lleva a considerar tomar acciones prontas, más certeras, que impacten a las personas adolescentes. En la investigación anterior, elaborada en el 2013, en la GAM, el 13\% tenía deseos de morir y el 8\% había pensado un plan para ejecutarlo, lo cual refleja que los datos se mantienen para las dos regiones estudiadas. A su vez, en la encuesta del 2006, el 31\% indicó que tenía deseos de morir.

Estos datos demuestran que hace diez años, las cifras de adolescentes que deseaban morir eran muy significativas, generando gran responsabilidad a los sectores de salud pública por buscar una solución al problema. Con el paso del tiempo se ha logrado notar, a partir de los resultados actuales, que el porcentaje ha disminuido en un $20 \%$, pero aun así no dejan de ser alarmantes y motivan a continuar buscando métodos de abordaje más eficaces.

De las personas adolescentes encuestadas, el $42 \%$ participa en grupos organizados, de estos, el $40 \%$ participa en grupos religiosos y un $52 \%$ en deportivos; se observa un promedio del $14 \%$ en grupos culturales y un $6 \%$ en grupos comunitarios.

De las personas adolescentes encuestadas que participaron en grupos organizados, es claro que aquellos religiosos y deportivos son fuertes movimientos grupales que impactan la vida de los jóvenes de forma positiva, usualmente, consolidando creencias, principios y valores espirituales, así como otras características personales que promueven estilos de vida más saludables (Soler et al., 2015). 
Estos datos mantienen una constancia a lo largo del tiempo, según lo observado en las encuestas del 2006 y 2013, razón que fortalece la creencia de continuar creando espacios sanos, recreativos y culturales para contribuir al crecimiento y al desarrollo integral del adolescente.

En relación con las horas utilizadas en herramientas tecnológicas, se evidenció que el $57 \%$ ve televisión un promedio de 1 a 3 horas, el $15 \%$ de 4 a 6 horas y el $12 \%$ más de 6 horas diarias. Un 13\% reportó no ver televisión.

La Academia Americana de Pediatría (AAP) recomienda que las personas adolescentes establezcan "zonas libres de pantallas" en el hogar, asegurándose de que no hay televisión, computadora o videojuegos en los dormitorios de los niños, y apagando la televisión durante las comidas. Las personas menores de edad deberían usar estos medios durante no más de una o dos horas diarias, y siempre con contenidos de alta calidad. Según lo reflejado en este estudio, el promedio de horas que los jóvenes invierten sería de 84 horas al mes, dato que confronta la realidad de la falta de tiempo de esparcimiento y recreación que se recomienda (APP citado en Soler, 2015).

En cuanto al uso de videojuegos, el $28 \%$ los utiliza de 1 a 3 horas, el $8 \%$ de 4 a 6 horas y un $9 \%$ más de 6 horas diarias. Un 51\% reporta no jugar videojuegos.

Por último, el $41 \%$ utiliza internet de 1 a 3 horas, el $15 \%$ de 4 a 6 , un $24 \%$ más de 6 horas diarias y el $17 \%$ reporta no tener acceso.

Se considera la posibilidad de que estos datos estén sesgados, ya que las personas adolescentes pueden no estar contabilizando el tiempo invertido, jugando en los celulares, los cuales se valoran solo como un medio de comunicación. Se recalca que estos datos son significativamente menores en comparación con los resultados del 2006 de la GAM, donde un 61\% reporta un uso de 1 a 3 horas, un $13 \%$ de 4 a 6; cuando mayor de 6 horas, los porcentajes son similares ( $8 \%$ ).

La disminución en los datos actuales, puede deberse a diversos factores, los cuales han de ser valorados de acuerdo con las dinámicas de los niños y adolescentes en la actualidad, en función de padres y el estilo de vida que llevan.

\section{Conclusiones y recomendaciones}

El análisis de los resultados de la encuesta permite concluir elementos importantes en cuanto a las intervenciones necesarias con las personas adolescentes en los niveles individual, familiar, escolar y social. Entre ellos, destaca establecer mecanismos de comunicación efectivos, que permitan brindar información y formación con respecto a los temas explorados en la encuesta.

Si se comparan los datos de la encuesta realizados por Chaves, Morales, Ramírez, Rodríguez, Sevilla, Ureña y Yock (2006), se nota cómo, de manera ponderada con la muestra, no han variado de significativamente los conocimientos, en particular los que se definen como protectores.

Uno de los temas que evidencia lo anterior es sexualidad, pues hay mejorías en el conocimiento de algunos aspectos, empero se siguen dando vacíos muy importantes y verdaderamente significativos, los que constituyen factores de riesgo para relaciones de violencia, embarazos tempranos y contagio de infecciones de transmisión sexual.

Sin embargo, el hecho de que el inicio de la actividad sexual coital en esta muestra es bajo, reconfirma la posibilidad de ejecutar acciones de prevención viables en la secundaria y que la escolarización per se es un factor protector para el inicio de actividad sexual coital temprana y desprotegida. 
En relación con la exposición a la pornografía, se concluye que es una conducta de riesgo importante, sumado a que los jóvenes cada vez tienen menos control de parte de sus padres, pasan mucho tiempo solos, y con la tecnología a su alcance en forma ilimitada y sin censura, sin duda aumenta las posibilidades de "entretenimiento con la pornografía". En la atención de adolescentes que cometen ofensas sexuales contra personas menores de edad en la Clínica de Adolescentes del Hospital Nacional de Niños, se ha encontrado que el inicio temprano de exposición a este material es un factor de alto riesgo para conductas sexuales abusivas.

El uso de la pornografía pareciera estar asociado con las actitudes sexuales permisivas $\mathrm{y}$ tiende a vincularse con las fuertes creencias de estereotipos sexuales en géneros. También se relaciona con las experiencias mayores de comportamiento sexual, con agresión sexual, ambas en términos de perpetración y victimización.

En cuanto al tema de la violencia, es de tomar en cuenta que los adolescentes están reconociendo diferentes tipos de agresión, incluida la emocional. Si contraponemos lo anterior con la información que tiene esta población, de si los agresores usualmente son conocidos o desconocidos, un número elevado menciona que en la mayoría de los casos son desconocidos, lo cual se contradice con los datos del país y de esta misma investigación.

Precisamente, en la población que reconoció haber sido víctima de violencia, queda de manifiesto que son los conocidos quienes cometen más agresiones e incluso el lugar donde más se dan estas situaciones es la casa de las víctimas, que muchas veces comparten con sus ofensores. Esta combinación de factores atenta contra la prevención y puede estar poniendo de manifiesto que el fenómeno de la violencia está presente en las familias y manteniéndose bajo secreto, con el riesgo que conlleva.

Otra forma de violencia que se ha visibilizado en los últimos años y que ya los adolescentes identifican como tal es el bullying, de ahí la necesidad de continuar profundizando en las medidas necesarias para reducir situaciones de este tipo en centros educativos, que asocia fuertemente a conductas autodestructivas en las víctimas y de perpetuación de la violencia en los agresores.

Dentro de este mismo tema, los porcentajes significativos de quienes han llevado armas al colegio y en la calle, muestra como estas prácticas son cada vez más frecuentes entre los y las adolescentes, lo que guarda relación directa con lo que está ocurriendo en la sociedad.

Sobre el tema del uso o consumo de drogas, los datos denotan un aumento considerable, con un inicio temprano de consumo de tabaco, alcohol y otras drogas, el cual se incrementa con la edad y el nivel académico. En los últimos años escolares (décimo y undécimo), una gran mayoría de las personas adolescentes encuestadas ha consumido alcohol con un porcentaje significativo de embriaguez. En el mismo sentido, ha aumentado el consumo de drogas ilegales, siendo la marihuana la que muestra un crecimiento significativo, muy probablemente asociado a que se concibe como "natural", lo que es equivalente a inocua, cuando la realidad es que ha tenido un incremento alarmante el número de personas jóvenes internadas en el Hospital Nacional Psiquiátrico por cuadros mentales asociados al consumo de esta droga.

En cuanto al tema de la alimentación, se encontró que un número significativo de adolescentes dice estar comiendo acompañado, sin embargo, no se puede afirmar la calidad de estos espacios; sería esperanzador que pudieran 
darse en un marco de convivencia de adultos y adolescentes, donde estos últimos pudieran expresar sentimientos y pensamientos de manera que sean escuchados/as y motivados/as, y a la vez, desde el mundo adulto, fueran espacios de supervisión y prevención de conductas de riesgo.

Por otro lado, asociado al aumento en el diagnóstico de trastornos alimentarios preocupa que el $38,8 \%$ de las personas encuestadas dice tener como costumbre leer revistas de moda y belleza, y un $29,67 \%$ de adolescentes ha efectuado algún tipo de dieta para bajar de peso, ambos factores de riesgo identificados en esta patología. En su mayoría son mujeres quienes acostumbran a leer este material, marcando la diferencia en cuanto a exigencias de género y su impacto en la preocupación por el aspecto físico.

Otro aspecto fundamental, documentado en la investigación, son los hábitos alimentarios de riesgo, como, por ejemplo, que el $98 \%$ de los encuestados consume comida rápida o chatarra al menos cada quince días, mostrando una de las razones que explicaría el aumento de problemas de salud en adolescentes, propio de los adultos, como obesidad, síndrome metabólico, diabetes mellitus tipo 2, hipertensión arterial, dislipidemias, entre otros.

Respecto a otros factores protectores, como son la participación en grupos que brindan contención, capacitación y opciones de participación social, se encontró un debilitamiento de la incorporación a estos.

En la investigación, los porcentajes señalan que los grupos religiosos van perdiendo protagonismo y los adolescentes tienen cada vez menos posibilidades de incorporarse a agrupaciones de iguales y de participación social.

Al analizar el uso de las herramientas de internet, como las redes sociales, estos se convierten en una posible estrategia de promoción y prevención, para divulgar la información y aclarar dudas y consultas, dado que un porcentaje mayoritario tiene acceso a estas herramientas y se han convertido en medios de comunicación de uso cotidiano para esta población.

Los resultados obtenidos demuestran la presencia de carencias protectoras significativas ante amenazas más complejas y que requieren del interés de la sociedad en su conjunto de la población adolescente.

Un buen ejemplo de la desatención de este grupo poblacional es el acceso a servicios de salud, puesto que la cobertura es de apenas de un 30\% en la Caja Costarricense de Seguro Social (CCSS), invisibilizando sus necesidades, al no contar con posibilidades de acudir a consultas diferenciadas.

Finalmente, esta investigación viene a reafirmar las debilidades y las deudas que como sociedad se tienen con la población adolescente y la necesidad de:

1- reformular el modelo de educación que permita una menor expulsión escolar y favorezca el concepto de comunidad educativa, en donde los padres y madres tengan un involucramiento activo, y la población adolescente tenga opciones más allá de lo académico para favorecer un desarrollo integral y que se dé una efectiva contención;

2- mantener, fortalecer y profundizar el programa de Educación en Sexualidad y Afectividad del Ministerio de Educación Pública, extendiéndolo a quinto y sexto grado de escuela como estaba previsto originalmente. En esta investigación, nuevamente se pone de manifiesto que el porcentaje de adolescentes escolarizados y menores de 17 años que inician actividad sexual coital es bajo. Lo anterior da un margen muy significativo para transmitir información protectora y, por lo tanto, de prevención efectiva; 
3- ahondar en los programas preventivos contra todas las formas de violencia, incluido el bullying, por parte del MEP;

4- plantear una lucha frontal desde el gobierno y en asocio con los padres y madres de familia contra el consumo de drogas, incluido el alcohol y el tabaco, tomando en cuenta todos los niveles de prevención y atención;

5- mantener y reforzar en el MEP las medidas encaminadas a mantener la norma de contar con alimentación sana en las sodas escolares y de enfatizar en los padres y madres de familia la importancia de preparar meriendas saludables para sus hijos e hijas;

6- promover y divulgar en los padres y madres fijarse, como meta protectora, mantener comidas familiares diarias que permitan un espacio de diálogo, contención y supervisión espontáneo con sus hijos e hijas;

7- garantizar el acceso a comida saludable a la población infantil y adolescente, regulando la venta de comidas rápidas, particularmente las de franquicias internacionales, prohibiendo ofertas especiales, ya sea que ofrezcan más cantidad de producto por un menor precio o que ofrezcan regalos de figuras infantiles; además, debe obligarse al etiquetado vistoso en los empaques con la información de aporte calórico de cada producto; que se restrinja el consumo de bebidas azucaradas (refill), que haya una mayor disposición de frutas y ensaladas y se plantee un impuesto adicional, similar al del tabaco, a este tipo de productos;

8- capacitar a los padres, madres de familia y educadores en la prevención y detección temprana de los trastornos de la alimentación, prioritariamente anorexia, bulimia y obesidad;

9- fortalecer políticas para recuperar espacios seguros para adolescentes y jóvenes, los cuales promuevan y faciliten actividades culturales, deportivas, recreativas y de participación social;
10- desarrollar estrategias de información y consulta para adolescentes en internet, integrando los sectores salud, educación y el Patronato Nacional de la Infancia;

11- reactivar, prioritariamente, un programa en salud desde la CCSS, diferenciado y amigable para la atención de adolescentes y jóvenes de 12 a 25 años.

\section{Referencias}

Chaves, R., Morales, A., Ramírez, W., Rodríguez, M., Sevilla, A., Ureña, M., y Yock, I. (2006). Situación actual de la adolescencia en la educación secundaria en Costa Rica: conocimientos, prácticas y protección. Clínica de Adolescentes, Hospital Nacional de Niños. Versión digital.

Hernández, M., Sánchez, D., Cañón, Y., Rojas, J., Aeón, A., y Santos, M. (2013) Factores de riesgo psicosocial que inciden en el consumo de sustancias psicoactivas en los adolescentes. Revista Línea de Vida 1(2013), 24-32. Recuperado de http://revia.areandina.edu.co/ojs/index. $\mathrm{php} / \mathrm{Kk} /$ article/view/372

Instituto de Alcoholismo y Farmacodependencia (2015). Encuesta Nacional sobre consumo de drogas en población de Educación Secundaria, Costa Rica. Recuperado de: http://www.iafa.go.cr/ investigaciones/epidemiologica/Consumo drogas \%20juventud escolarizada 2012.pdf

Organización Mundial de la Salud (2002). Informe mundial sobre la violencia y la salud. Washington, DC: OPS. Recuperado de: http://psiquiatria.facmed.unam.mx/doctos/descargas/ Violencia\%20OMS\%5B1\%5D\%5B1\%5D.pdf

Organización Mundial de la Salud (2013). Comprender y abordar la violencia contra las mujeres. Recuperado de: http://apps.who. int/iris/bitstream/handle/10665/98821/ WHO RHR 12.37 spa.pdf;jsessioni$\mathrm{d}=\mathrm{E} 316$ A573957AC35BE41FC779651E1CD$\mathrm{F}$ ? sequence $=1$ 
Organización Mundial de la Salud (2016). Salud sexual. Recuperado de: http://www.who.int/ topics/sexual health/es/

Rivera, R., y Chuana Cuentas, M. (2016). Influencia de la familia sobre conductas antisociales en adolescentes de Arequipa- Perú. Actualidades en Psicología, 30 (120), 85-97.: ISSN 22153535

Soler, A. (2015). Los niños y la exposición a la televisión, móviles y tablets: ¿Qué dice la ciencia?
Recuperado de http://www.albertosoler.es/ los-ninos-y-la-exposicion-a-television-moviles-y-tablets-que-dice-la-ciencia/

Soler, M., Pérez, E., Mejías., M., López, C., Lesar, M. (2015). Programa educativo para potenciar factores de riesgo en adolescentes con conducta suicida. Revista Médica Multi Med,. 20 (1), 161-171. Recuperado de http://www. revmultimed.sld.cu/index.php/mtm/article/ view/139/135 